\title{
Quotients over Minimal Type Theory
}

\author{
Maria Emilia Maietti \\ Dipartimento di Matematica \\ Università di Genova, Italy \\ maietti@math.unipd.it
}

\begin{abstract}
We consider an extensional version, called qmTT, of the intensional Minimal Type Theory mTT, introduced in a previous paper with G. Sambin, enriched with proof-irrelevance of propositions and effective quotient sets. Then, by using the construction of total setoid à la Bishop we build a model of qmTT over mTT.

The design of an extensional type theory with quotients and its interpretation in mTT is a key technical step in order to build a two level system to serve as a minimal foundation for constructive mathematics as advocated in the mentioned paper about mTT.
\end{abstract}

Keywords: Dependent type theory, intuitionistic logic, quotient completion.

\section{Introduction}

In [MS05] we argued for the need of a minimal foundation for constructive mathematics. We wanted this theory to be minimal among relevant constructive foundations such as the generic internal theory of a topos and Martin-Löf's type theory, besides the classical Zermelo-Fraenkel set theory. Then, being Martin-Löf's type theory predicative, our theory must be predicative, too.

The constructivity of our foundation is expressed by the fact that it satisfies the proofs-as-programs paradigm. In [MS05] we motivated that an essential characteristic of a proofs-as-programs theory must be its consistency with the axiom of choice and the formal Church thesis altogether. In other words, the proofs-as-programs theory must be equipped with a realizability model where the extraction of programs from proofs is internalized by validating the axiom of choice and the formal Church thesis as internal theorems.

But, then, it turned out that this requirement is so strong to be incompatible with another desirable feature that a foundation for mathematics should have, namely with the capability of representing extensional concepts as those used in everyday mathematics. In other terms, as reported in [MS05], we cannot have an extensional theory that is also proofs-as-programs in our sense. Indeed, it is wellknown that extensionality of functions is inconsistent with the axiom of choice and the formal Church thesis altogether. And, for an extensional constructive theory even the consistency with the axiom of choice alone can be a problem. Indeed, while the axiom of choice is an accepted principle in intensional type 
theory, it is not generally validated in an extensional constructive one since it may force the theory to be classical (see for example [Mai99], [MV99], [Car04], [ML06]). This is simply because the choice function cannot be always guaranteed to be extensional constructively.

The solution proposed in [MS05] to the problem of building an extensional proofs-as-programs foundation consists in building a two level theory: one should start with an intensional proofs-as-programs theory in the above sense and then build an extensional level upon it according to the forget-restore principle in [SV98]. This principle says that extensional concepts must be designed by abstracting on the intension of their representations at the intensional level in such a way that all the forgotten computational information of their representations can be restored at will. An example of this is the design of the many-sorted logic obtained from a type theory by using Martin-Löf's true-judgements (see [Mar84], [SV98]). In this way proofs at the extensional level are turned into proofs at the intensional level that correspond to programs. We then decided to name programs level the intensional one and proofs level the extensional one to express that the link between the two levels is also part of the proofs-as-programs transformation.

To serve as the intensional level of the minimal foundation advocated in [MS05], we there introduced Minimal Type Theory (mTT). This is obtained by extending the set constructors of intensional Martin-Löf's type theory in [NPS90] with a primitive notion of propositions. The main difference between our theory and Martin-Löf's one is that the axiom of choice and even the axiom of unique choice are not valid theorems in $\mathrm{mTT}$.

Here we assume the extensional level to be given by a type theory, called qmTT. This is obtained as follows. First, we take the extensional version of our Minimal Type Theory, in the same way as the type theory in [Mar84] is the extensional version of intensional Martin-Löf's type theory in [NPS90]. Then we collapse propositions into mono sets in the sense of [Mai05] and, finally, we add effective quotient sets similarly to those in [Mai05].

In order to interpret our extensional type theory qmTT in mTT we build a category $\mathrm{Q}(\mathrm{mTT})$ of total setoids, whose objects and morphisms coincide with the notion of sets and functions given by E. Bishop [Bis67].

$\mathrm{Q}(\mathrm{mTT})$ turns out to be a categorical model of qmTT. Categorically speaking, it turns out to be a lextensive list-arithmetic locally cartesian closed category with stable effective quotients of equivalence relations obtained by comprehension from a propositional fibration (for all these categorical properties see, for example, [Jac99,Mai05]).

Our total setoid model corresponds categorically to a different quotient completion from the same construction of total setoids performed over Martin-Löf's type theory [NPS90], here called MLTT, as studied in [Pal05]. In fact, the total setoid model Q(MLTT) over MLTT coincides with the exact completion of the weakly lex category [CV98,CC82] of the MLTT sets. Instead, Q(mTT) can be seen as an instance of a more general completion of quotients starting from a weakly cartesian category equipped with a suitable comprehensive fibration. 
It is worth noting that in both models there are at least two ways of interpreting propositions. One consists in interpreting propositions as sets. Then, both in $\mathrm{Q}(\mathrm{mTT})$ and in $\mathrm{Q}(\mathrm{MLTT})$ the extensional version of Martin-Löf's type theory in [Mar84] is validated. Therefore, the axiom of choice where quantifiers are interpreted as dependent product and indexed sum is also valid in both. But then we know that the axiom of choice may be constructively incompatible with well-behaved quotients, in particular effectiveness of quotients (see [Mai99]). Therefore, it seems that our total setoid constructions cannot be considered as a quotient completion of a propositions-as-sets theory.

The other way of interpreting propositions consists in interpreting them in $\mathrm{Q}(\mathrm{MLTT})$ as all mono sets (as in [Mai05]), and in Q(mTT) as only some mono sets like in qmTT. Then, both models support well-behaved quotients, i.e. effective quotients. In particular, the internal language of Q(MLTT) includes that of locally cartesian closed pretopos in [Mai05]. But, by interpreting propositions as mono sets, $\mathrm{Q}(\mathrm{MLTT})$ and even more $\mathrm{Q}(\mathrm{mTT})$ loose the general validity of the propositional axiom of choice. In fact, following propositions as mono sets, the interpretation of the axiom of choice in Q(MLTT) turns out to be equivalent to what Martin-Löf calls the extensional axiom of choice in [ML06], known to fail constructively [ML06,Car04]. Only the validity of the axiom of unique choice survives in its generality in Q(MLTT).

The design of qmTT and its interpretation over mTT is a key technical step in order to build the extensional level of the minimal constructive foundation advocated in [MS05]. This extensional level does not exactly coincide with qmTT, since qmTT is just obtained from mTT by abstracting on the intensional equality between elements of sets and propositions. Further abstractions, like that from proof-terms of propositions or the addition of subsets, are also desirable in order to get a many-sorted logic closer to set-theoretic languages used in everyday mathematics. However these can be obtained by associating to qmTT a many sorted logic with true-judgements as in [Mar84,Mar85] and subsets as in [SV98,Car03]. Another important point is that the extensional level advocated in [MS05] should have predicates depending on more general types than sets, like the collection of all subsets of a set (these are particularly needed in a predicative theory to define some of common mathematical concepts, like, for example, the definition of formal topology in [Sam03]).

Here we decided to concentrate on how to interpret quotients in mTT given that various proposals of how to add quotients have been given in the literature of type theory with the notion of setoid (see for example [Hof97] and [BCP03] and references therein) and also in category theory with the notion of quotient completions of a (weakly) lex category or of a regular one (see for example [CC82,CV98,Car95,CR00]). The exact formulation of the extensional level of the minimal constructive foundation based on mTT is left to future work with G. Sambin. 


\section{The extensional system}

Here we briefly introduce the extensional type theory qmTT that we will interpret in a model built out of the intensional type theory mTT introduced in [MS05]. We assume that mTT includes also a boolean universe (as mentioned in [Car04] and whose rules are the same as those for $U_{b}$ of qmTT in the appendix) to make the disjoint sum set really disjoint (see, for example, [Mai05] for its definition).

qmTT is obtained as follows: we first take the extensional version of $\mathrm{mTT}$, in the same way as Martin-Löf's type theory in [Mar84] is the extensional version of that in [NPS90]; then we collapse propositions into mono sets according to the notion in [Mai05]; and finally we add effective quotient sets as in [Mai05]. The precise rules to form sets and propositions of qmTT are given in the appendix.

The form of judgements to describe qmTT are those of mTT. Hence, for building sets, in the style of Martin-Löf's type theory [Mar84,NPS90], we have four kinds of judgements:

$$
A \text { set }[\Gamma] \quad A=B[\Gamma] \quad a \in A[\Gamma] \quad a=b \in A[\Gamma]
$$

that is the set judgement, the equality between sets, the term judgement and the (definitional) equality between terms of the same set, respectively. The contexts $\Gamma$ of these judgements are formed as in [Mar84] and they are telescopic [dB91] since sets are allowed to depend on variables ranging over other sets.

The set constructors of qmTT are those of mTT with the addition of effective quotient sets (see [Mai05]).

Moreover, to build propositions, as in $\mathrm{mTT}$, we have the following judgements:

$$
A \text { prop }[\Gamma] \quad A=B[\Gamma]
$$

In order to make propositions into mono sets, namely to make propositions into sets inhabited with at most one proof according to the notion in [Mai05], we add the rules

$$
\text { prop-into-set } \frac{A \text { prop }[\Gamma]}{A \operatorname{set}[\Gamma]} \quad \text { prop-mono } \frac{A \text { prop }[\Gamma] \quad p \in A[\Gamma] \quad q \in A[\Gamma]}{p=q \in A[\Gamma]}
$$

The requirement that propositions are mono sets is crucial in the presence of quotient effectiveness which would otherwise become similar to a choice operator. Indeed, if we identify propositions with sets simply, quotient effectiveness may lead to classical logic (see [Mai99]) and hence it is no longer a constructive rule. The propositions of qmTT are those of mTT but their proofs are all made equal. Moreover, the intensional propositional equality is replaced by the extensional one of [Mar84], which, besides being mono by definition, is equivalent to the definitional equality of terms.

Our extensional theory is a variation of the internal type theory of a lextensive list-arithmetic locally cartesian closed pretopos, as devised in [Mai05]. The main difference is that we discharge the identification propositions as mono sets typical of a pretopos by simply taking propositions as primitive mono sets, without requiring that all mono sets are propositions. In this way we avoid the validity 
of the axiom of unique choice, which would instead follow under the identification of propositions with mono sets (see [Mai05]).

The mono condition for propositions makes their proof-terms irrelevant. The proof-irrelevance of propositions is helpful to implement subsets as in [SV98] without the restrictions pointed out in [Car03].

\section{The setoid model}

We define the following category of sets equipped with an equivalence relation, sometimes called "total setoids" in the literature:

Definition 1. The category $\mathbf{Q}(\mathbf{m T T})$ is defined as follows:

ObQ(mTT): the objects are pairs $\left(A,=_{A}\right)$ where $A$ is a set in mTT, called "support", and

$$
x={ }_{A} y \text { prop }[x \in A, y \in A]
$$

is an equivalence relation on the set $A$. This means that in mTT there exist proof-terms witnessing reflexivity, symmetry and transitivity of the relation:

$$
\begin{aligned}
& \operatorname{refl}(x) \in x={ }_{A} x \quad[x \in A] \\
& \operatorname{sym}(x, y, z) \in y={ }_{A} x \quad\left[x \in A, y \in A, z \in x={ }_{A} y\right] \\
& \operatorname{trans}(x, y, z, u, v) \in x={ }_{A} z \quad\left[x \in A, y \in A, z \in A, u \in x={ }_{A} y, v \in y={ }_{A} z\right]
\end{aligned}
$$

$\operatorname{MorQ}(\mathbf{m T T})$ : the morphisms from an object $\left(A,={ }_{A}\right)$ to $\left(B,=_{B}\right)$ are terms $f(x) \in B[x \in A]$ preserving the corresponding equality, i.e. in mTT there exists a proof-term

$$
\operatorname{pr}_{1}(x, y, z) \in f(x)={ }_{B} f(y) \quad\left[x \in A, y \in A, z \in x={ }_{A} y\right]
$$

Moreover, two morphisms $f, g:\left(A,={ }_{A}\right) \rightarrow\left(B,={ }_{B}\right)$ are equal if and only if in mTT there exists a proof-term

$$
\operatorname{pr}_{2}(x) \in f(x)={ }_{B} g(x) \quad[x \in A]
$$

This category comes naturally equipped with an indexed category (or fibration) satisfying comprehension (see [Jac99] for its definition):

Definition 2. The indexed category:

$$
\mathcal{P}_{q}: \mathrm{Q}(\mathrm{mTT})^{\mathrm{OP}} \rightarrow \text { Cat }
$$

is defined as follows. For each object $\left(A,=_{A}\right)$ in $\mathrm{Q}(\mathrm{mTT})$ then $\mathcal{P}_{q}\left(\left(A,=_{A}\right)\right)$ is the following category: $\operatorname{ObP}_{q}\left(\left(A,=_{A}\right)\right)$ are the propositions $\phi(x)$ prop $[x \in A]$ depending on $A$ and preserving the equality on $A$, namely there exists a proofterm:

$$
\operatorname{ps}(x, y, d) \in \phi(x) \rightarrow \phi(y)\left[x \in A, y \in A, d \in x={ }_{A} y\right]^{1}
$$

and two propositions are equal if they are equiprovable in $\mathrm{mTT}$.

\footnotetext{
${ }^{1}$ Indeed, from this, by using the symmetry of $x={ }_{A} y$ it follows that $\phi(x)$ is equivalent to $\phi(y)$ if $x={ }_{A} y$ holds.
} 
Morphisms in $\operatorname{Mor}_{q}\left(\left(A,=_{A}\right)\right)$ are given by a partial order, namely

$$
\begin{aligned}
& \mathcal{P}_{q}\left(\left(A,=_{A}\right)\right)(\phi(x), \psi(x)) \equiv \phi(x) \leq \psi(x) \\
& \quad \text { iff there exists a proof-term } \operatorname{pt}(x) \in \phi(x) \rightarrow \psi(x)[x \in A]
\end{aligned}
$$

Moreover, for every morphism $f:\left(A,={ }_{A}\right) \rightarrow\left(B,=_{B}\right)$ in $Q(m T T)$ given by $f(x) \in B[x \in A]$ then $\mathcal{P}_{q}(f)$ is the substitution functor, i.e. $\mathcal{P}_{q}(f)(\phi(y)) \equiv$ $\phi(f(x))$ for any proposition $\phi(y)$ prop $[y \in B]$ (recall that $\mathcal{P}_{q}$ is contravariant).

Lemma 1. $\mathcal{P}_{q}$ is an indexed category satisfying the universal property of comprehension.

Proof. To show the property of comprehension, we consider the Grothendieck completion $\operatorname{Gr}\left(\mathcal{P}_{q}\right)$ of $\mathcal{P}_{q}$ (see [Jac99] for its definition) and the functor $T$ : $\mathrm{Q}(\mathrm{mTT}) \rightarrow \operatorname{Gr}\left(\mathcal{P}_{q}\right)$ defined as follows:

$$
T\left(\left(A,=_{A}\right)\right) \equiv\left(\left(A,=_{A}\right), \mathrm{tt}\right) \quad T(f) \equiv\left(f, i d_{\mathrm{tt}}\right)
$$

where tt is the truth constant that can be represented by any tautology.

$T$ satisfies the following universal property of comprehension (cfr. [Jac99]): for every $\operatorname{Gr}\left(\mathcal{P}_{q}\right)$-object $\left(\left(A,=_{A}\right), P\right)$ there exists a $\mathrm{Q}(\mathrm{mTT})$-object

$$
C m\left(\left(\left(A,=_{A}\right), P\right)\right) \equiv\left(\Sigma_{x \in A} P(x),=_{C m}\right)
$$

where $z_{1}={ }_{C m} z_{2} \equiv \pi_{1}\left(z_{1}\right)={ }_{A} \pi_{1}\left(z_{2}\right)$ for $z_{1}, z_{2} \in \Sigma_{x \in A} P(x)$, such that, for each $\operatorname{Gr}\left(\mathcal{P}_{q}\right)$-morphism $(f, p): T\left(\left(C,=_{C}\right)\right) \longrightarrow\left(\left(A,=_{A}\right), P\right)$ there is a unique morphism $[f, p]:\left(C,=_{C}\right) \longrightarrow C m\left(\left(\left(A,=_{A}\right), P\right)\right)$ in $\mathrm{Q}(\mathrm{mTT})$ such that in $\operatorname{Gr}\left(\mathcal{P}_{q}\right)$

$$
\left(\eta_{1}, \eta_{2}\right) \cdot T([f, p])=(f, p)
$$

where $\eta_{1} \equiv \pi_{1}^{P}$ and $\eta_{2} \equiv \pi_{2}^{P}$ are the first and second projections of the indexed $\operatorname{sum} \Sigma_{x \in A} P(x)$.

Definition 3 ( $\mathcal{P}_{q}$-equivalence relations). Given an equivalence relation $R \in$ $\mathcal{P}_{q}\left(\left(A,=_{A}\right) \times\left(A,=_{A}\right)\right)$, namely a predicate $R(x, y)$ prop $[x \in A, y \in A]$ that preserves $={ }_{A}$ on both dependencies and is also an equivalence relation, then the first component of the counit on $\left(\left(A,=_{A}\right) \times\left(A,=_{A}\right), R\right)$ is a monic equivalence relation in $\mathrm{Q}(\mathrm{mTT})$, and it is called a $\mathcal{P}_{q}$-equivalence relation.

The category $\mathrm{Q}(\mathrm{mTT})$ enjoys all the categorical properties necessary to interpret qmTT (for their definitions see, for example, [Mai05]).

Theorem 1. The category $\mathrm{Q}(\mathrm{mTT})$ is lextensive (i.e. with terminal object, binary products, equalizers and stable finite disjoint coproducts) list-arithmetic (i.e. with parameterized lists) and locally cartesian closed (i.e. with also dependent products) with stable effective quotients with respect to $\mathcal{P}_{q}$-equivalence relations. Moreover, the indexed category $\mathcal{P}_{q}$ validates first-order intuitionistic logic. 
Remark 1. Note that to prove theorem 1 is crucial to have explicit proof-terms witnessing that $x={ }_{A} y$ in a $\mathrm{Q}(\mathrm{mTT})$-object is an equivalence relation, that a $\mathrm{Q}(\mathrm{mTT})$-morphism preserves the corresponding equivalence relations and when two $\mathrm{Q}(\mathrm{mTT})$-morphisms are equal. In other words it seems that it would not follow if we give the definition of setoid objects, morphisms and their equality via true-judgements of the kind $x={ }_{A} y$ true $[x \in A, y \in A]$ as in the first setoid model in [Hof97] ${ }^{2}$.

The interpretation of qmTT in $\mathbf{Q}(\mathbf{m T T})$. After theorem 1, in order to interpret qmTT in $\mathrm{Q}(\mathrm{mTT})$ we could adapt the interpretation in [Mai05] given by fibred functors (we recall that this overcomes the problem, first solved in [Hof94], of interpreting substitution correctly when following the informal interpretation first given by Seely in [See83] and recalled in [Joh02]). But this interpretation requires a choice of the structure and is not first order, since it requires to quantify over fibred functors. Luckily, in our case we can give a predicative interpretation in the setoid model similar to that in the completeness proof in [Mai05] (by using coherent isomorphisms!). This is because the setoid model is indeed a syntactic one!

The key point to get this interpretation is to note that the slice category of arrows in $\left(A,=_{A}\right)$ is equivalent to the category of dependent setoids as defined in [Bis67,Pal05].

Definition 4. Given an object $\left(A,=_{A}\right)$ of $\mathrm{Q}(\mathrm{mTT})$, abbreviated with $A_{=}$, we define a dependent setoid on the setoid $\left(A,={ }_{A}\right)$ written

$$
B=(x)\left[x \in A_{=}\right]
$$

as a dependent set $B(x)$ set $[x \in A]$, called "dependent support", together with an equivalence relation

$$
y={ }_{B(x)} y^{\prime} \text { prop }\left[x \in A, y \in B(x), y^{\prime} \in B(x)\right] .
$$

Moreover, for any $x_{1}, x_{2} \in A$ there must exist

$$
\sigma_{x_{1}, x_{2}}(y) \in B\left(x_{2}\right)\left[x_{1} \in A, x_{2} \in A, d \in x_{1}={ }_{A} x_{2}, y \in B\left(x_{1}\right)\right]
$$

non depending on $d \in x_{1}={ }_{A} x_{2}$ and preserving the equality on $B\left(x_{1}\right)$, namely there exists a proof of

$$
\begin{aligned}
\sigma_{x_{1}, x_{2}}(y)={ }_{B\left(x_{2}\right)} \sigma_{x_{1}, x_{2}}\left(y^{\prime}\right) \text { prop }\left[x_{1}\right. & \in A, x_{2} \in A, d \in x_{1}={ }_{A} x_{2}, \\
y & \left.\in B\left(x_{1}\right), y^{\prime} \in B\left(x_{1}\right), w \in y={ }_{B\left(x_{1}\right)} y^{\prime}\right] .
\end{aligned}
$$

Furthermore, $\sigma_{x, x}$ is the identity, namely there exists a proof of

$$
\sigma_{x, x}(y)=_{B(x)} y \in B(x) \text { prop }[x \in A, y \in B(x)]
$$

\footnotetext{
${ }^{2}$ Note that the mentioned setoid model is anyway very different from $\mathrm{Q}(\mathrm{mTT})$ since the morphism equality is simply the definitional equality of the terms between the setoid supports.
} 
and the $\sigma_{x_{1}, x_{2}}$ 's are closed under composition, namely there exists a proof of

$$
\begin{aligned}
& \sigma_{x_{2}, x_{3}}\left(\sigma_{x_{1}, x_{2}}(y)\right)={ }_{B\left(x_{3}\right)} \sigma_{x_{1}, x_{3}}(y) \text { prop } \\
& \quad\left[x_{1} \in A, x_{2} \in A, x_{3} \in A, y \in B\left(x_{1}\right), d_{1} \in x_{1}={ }_{A} x_{2}, d_{2} \in x_{2}={ }_{A} x_{3}\right] .
\end{aligned}
$$

Categorically speaking, the category having the elements of $A$ with their equality as objects and $\sigma_{x_{1}, x_{2}}$ as (the unique) morphism from $x_{1}$ to $x_{2}$ forms a groupoid, because every $\sigma_{x_{1}, x_{2}}$ gives rise to an isomorphism between $B\left(x_{1}\right)$ and $B\left(x_{2}\right)$.

Definition 5. Let us call $\operatorname{Dep}\left(\left(A,=_{A}\right)\right)$ the category whose objects are dependent setoids $B_{=}(x)\left[x \in A_{=}\right]$on the setoid $\left(A,=_{A}\right)$, and whose morphisms

$$
b(x) \in B_{=}(x)\left[x \in A_{=}\right]
$$

are dependent terms $b(x) \in B(x)[x \in A]$ preserving the equality on $A$, namely in mTT there exists a proof of

$$
\sigma_{x_{1}, x_{2}}\left(b\left(x_{1}\right)\right)={ }_{B\left(x_{2}\right)} b\left(x_{2}\right) \text { prop }\left[x_{1} \in A, x_{2} \in A, d \in x_{1}={ }_{A} x_{2}\right] .
$$

Proposition 1. The category $\mathrm{Q}(\mathrm{mTT}) /\left(A,=_{A}\right)$ is equivalent to Dep $\left(\left(A,=_{A}\right)\right)$.

This proposition suggests that the categorical interpretation in $\mathrm{Q}(\mathrm{mTT})$ given in [Mai05] can be equivalently formulated by interpreting dependent sets into dependent setoids as follows.

Sketch of the interpretation of qmTT into dependent setoids: A dependent set $B\left(x_{1}, \ldots x_{n}\right)\left[x_{1} \in A_{1}, \ldots x_{n} \in A_{n}\right]$ of qmTT is interpreted as a dependent setoid

$$
B_{=}\left(x_{1}, \ldots x_{n}\right)^{I}\left[x_{1} \in A_{1=}^{I}, \ldots x_{n} \in A_{n=}^{I}\right]
$$

assuming to have generalized def. 4 to setoids with telescopic dependencies, where $B\left(x_{1}, \ldots x_{n}\right)^{I}\left[x_{1} \in A_{1}{ }^{I}, \ldots x_{n} \in A_{n}{ }^{I}\right]$ is its dependent support.

In the following, we leave the reader to deduce himself the $\sigma$ 's of the various dependent setoids. Except for the extensional propositional equality, any proposition is interpreted in the corresponding one of $\mathrm{mTT}$ with the warning that its equality is trivial, namely if $\phi$ is a proposition then $z={ }_{\phi^{I}} z^{\prime} \equiv$ tt for all $z, z^{\prime} \in \phi^{I}$. Hence, for example,

$$
(\phi \vee \psi)^{I} \equiv \phi^{I} \vee \psi^{I} \quad\left(\forall_{x \in B} C(x)\right)^{I} \equiv \forall_{x \in B^{I}} C^{I}(x) .
$$

Instead the extensional propositional equality is interpreted in the equality of the set to which it refers:

$$
\left(\operatorname{Eq}\left(B, b_{1}, b_{2}\right)\right)^{I} \equiv b_{1}^{I}={ }_{B^{I}} b_{2}^{I}
$$

The emptyset, the singleton and the boolean universe are interpreted in themselves with the equality given by the propositional one: for example

$$
\left(U_{b}\right)^{I} \equiv U_{b} \quad \text { and } \quad z=_{U_{b}^{I}} z^{\prime} \equiv \operatorname{ld}\left(U_{b}, z, z^{\prime}\right) \text { for } z, z^{\prime} \in U_{b} .
$$

Instead the other constructors are interpreted as follows: 
Strong Indexed Sum set : $\quad\left(\Sigma_{x \in B} C(x)\right)^{I} \equiv \Sigma_{x \in B^{I}} C^{I}(x)$

and $z=\sum_{\Sigma_{x \in B} C(x)^{I}} z^{\prime} \equiv \pi_{1}(z)={ }_{B^{I}} \pi_{1}\left(z^{\prime}\right) \wedge \sigma_{\pi_{1}(z), \pi_{1}\left(z^{\prime}\right)}\left(\pi_{2}(z)\right)==_{C^{I}\left(\pi_{1}\left(z^{\prime}\right)\right)} \pi_{2}\left(z^{\prime}\right)$ for $z, z^{\prime} \in \Sigma_{x \in B} C(x)^{I}$.

Disjoint Sum set : $\quad(B+C)^{I} \equiv B^{I}+C^{I}$ and $z={ }_{B^{I}+C^{I}} z^{\prime} \equiv\left\{\begin{array}{lll}b={ }_{B^{I}} b^{\prime} & \text { if } z=\operatorname{inl}(b) & z^{\prime}=\operatorname{inl}\left(b^{\prime}\right) \text { for } b, b^{\prime} \in B^{I} \\ c={ }_{C^{I}} c^{\prime} & \text { if } z=\operatorname{inr}(c) & z^{\prime}=\operatorname{inr}\left(c^{\prime}\right) \text { for } c, c^{\prime} \in C^{I} \\ \perp & \text { otherwise }\end{array}\right.$

for $z, z^{\prime} \in B^{I}+C^{I}$.

Dependent Product set : $\quad\left(\Pi_{x \in B} C(x)\right)^{I} \equiv$

$\Sigma_{h \in \Pi_{x \in B^{I}} C^{I}(w)} \quad \forall_{x_{1}, x_{2} \in B^{I}} x_{1}={ }_{B^{I}} x_{2} \rightarrow \sigma_{x_{1}, x_{2}}\left(h\left(x_{1}\right)\right)={ }_{C^{I}\left(x_{2}\right)} h\left(x_{2}\right)$

and $z={ }_{\Pi_{x \in B^{I}} C(x)^{I}} z^{\prime} \equiv \forall_{x \in B^{I}} \pi_{1}(z)(x)={ }_{C^{I}(x)} \pi_{1}\left(z^{\prime}\right)(x)$ for $z, z^{\prime} \in \Pi_{x \in B^{I}} C(x)^{I}$.

Quotient set : $(A / R)^{I} \equiv A^{I}$

and $z={ }_{A / R^{I}} z^{\prime} \equiv R^{I}\left(z, z^{\prime}\right)$ for $z, z^{\prime} \in A^{I}$.

List set : $\quad(\operatorname{List}(C))^{I} \equiv \operatorname{List}\left(C^{I}\right)$

and $z={ }_{\operatorname{List}(C)^{I}} z^{\prime} \equiv \exists_{l \in \operatorname{List}(R)} \operatorname{Id}\left(\operatorname{List}\left(C^{I}\right), \overline{\pi_{1}}(l), z\right) \wedge \operatorname{Id}\left(\operatorname{List}\left(C^{I}\right), \overline{\pi_{2}}(l), z^{\prime}\right)$

for $z, z^{\prime} \in \operatorname{List}(C)^{I}$ where $R \equiv \Sigma_{x \in C^{I}} \Sigma_{y \in C^{I}} x=_{C^{I}} y$ and $\overline{\pi_{i}} \equiv \operatorname{List}\left(\pi_{i}\right)$ is the lifting on lists of the $i$-th projection for $i=1,2$.

To see that the interpretation of disjoint sum sets is well-defined, recall that in mTT the sum is disjoint thanks to the presence of the boolean universe $U_{b}$.

Remark 2. Internal logic of $\mathbf{Q}(\mathbf{m} \mathbf{T T})$. We are not able to prove that $\mathrm{Q}(\mathrm{mTT})$ has qmTT as its internal language. The reason is that the interpretation of implication, of universal quantification and of dependent product set do not seem to be preserved by the functor $\xi: \mathrm{Q}(\mathrm{mTT}) \rightarrow \mathcal{C}(\mathrm{qmTT})$ sending a setoid into its quotient, where $\mathcal{C}(q \mathrm{mTT})$ is the syntactic category of qmTT defined as in [Mai05] (note that we can naturally interpret mTT into qmTT by sending all the constructors in the corresponding ones and, in particular, the intensional propositional equality of mTT into the extensional one of qmTT).

However, observe that the coherent fragment cqmTT of qmTT, obtained by cutting out implication, universal quantification and dependent product sets from qmTT, is the internal language of the setoid model built over the corresponding coherent fragment cmTT of mTT obtained by cutting out the corresponding sets and propositions.

Remark 3. Connection with the exact completion of a weakly lex category. The construction of total setoids on mTT corresponds categorically to an instance of the following generalization of the exact completion construction [CV98,CC82] of a weakly lex category: we start from a weakly cartesian category $\mathcal{C}$ endowed with a split comprehensive fibration $\mathcal{P}$ satisfying enough logical laws to express the notion of equivalence relation; then we simulate the exact completion of a weakly lex category by taking only those pseudo-equivalence relations coming by comprehension from equivalence relations in the fibres of 
$\mathcal{P}$. If $\mathcal{C}$ is weakly lex and $\mathcal{P}$ is the codomain fibration (see [Jac99]) then our construction is the exact completion of $\mathcal{C}$.

Therefore, knowing the internal language of such categorical constructions from [Mai05], we conclude that the total setoid construction coincides with the exact completion construction as in [CV98,CC82] if we perform such a construction on an extension of mTT, called MLTT, equivalent to Martin-Löf's type theory in [NPS90]. In order to get the the first order fragment of Martin-Löf's type theory it is enough to strengthen the existential quantifier of mTT to enjoy E- $\Sigma$ and C- $\Sigma$ of the Strong Indexed Sum set, after adding the rule that any set $A$ set is also a proposition $A$ prop (recall that in mTT propositions are not assumed to be proof-irrelevant!).

Then, the category Q(MLTT) of total setoids built on MLTT turns out to be a list-arithmetic locally cartesian closed pretopos and it coincides with the exact completion, as defined in [Car95,CV98], of the weakly lex category C(MLTT), where the category C(MLTT) is defined as follows: its objects are MLTT-sets and its morphisms from $A$ to $B$ are terms $b(x) \in B[x \in A]$ and two morphisms $b_{1}(x) \in B[x \in A]$ and $b_{2}(x) \in B[x \in A]$ are equal if there exists a proof of $\operatorname{ld}\left(B, b_{1}(x), b_{2}(x)\right)$ prop $[x \in A]$ in MLTT. The identity and composition are defined as in the syntactic categories in [Mai05].

However, it is important to note that the total setoid model Q(MLTT) does not seem to be closed under well-behaved quotients if we identify propositions as sets as done in MLTT. The reason is that under this identification Q(MLTT), but also $\mathrm{Q}(\mathrm{mTT})$, supports the axiom of choice where the quantifiers are replaced by $\Pi$ and $\Sigma$ as a consequence that they validate the extensional version of Martin-Löf's type theory in [Mar84]. Then, effectiveness of quotients, being generally incompatible with the axiom of choice (see [Mai99]), does not seem to be validated.

To gain well-behaved quotients in Q(MLTT) one possibility is to reason by identifying propositions as mono sets as in the logic of a pretopos (see [Mai05]). Instead, in $\mathrm{Q}(\mathrm{mTT})$ we get them by identifying propositions with only those mono sets arising from propositions in mTT as in the interpretation of qmTT. In fact, even if $\mathrm{Q}(\mathrm{mTT})$ supports quotients of all mono equivalence relations, these do not seem to enjoy effectiveness. Categorically speaking, this means that $\mathrm{Q}(\mathrm{mTT})$ does not seem to be a pretopos even if it has quotients for all monic equivalence relations because we are not able to prove that all monic equivalence relations are in bijection with $\mathcal{P}_{q}$-equivalence relations, for which effective quotients exist (which explains why we introduced the concept of $\mathcal{P}_{q^{-}}$ equivalence relation!).

As expected from [Mai99], under the identification propositions as mono sets, $\mathrm{Q}$ (MLTT) looses the validity of the axiom of choice. In fact it turns out that the propositional axiom of choice is exactly interpreted in Q(MLTT) (and also in $\mathrm{Q}(\mathrm{mTT}))$ as the extensional axiom of choice in [ML06,Car04] following the given interpretation of qmTT in $\mathrm{Q}(\mathrm{mTT})$. Therefore, the arguments in [ML06,Car04] exactly show that the propositional axiom of choice fails to be valid in the total setoid models $\mathrm{Q}(\mathrm{MLTT})$, and even more in $\mathrm{Q}(\mathrm{mTT})$, under the identification 
of propositions with mono sets. In Q(MLTT) the propositional axiom of choice survives only for those setoids whose equivalence relation is the propositional equality of MLTT. Only the validity of the axiom of unique choice continues to hold in its generality in Q(MLTT) (see also [ML06]).

Remark 4. In order to interpret quotients in mTT we also considered to mimic the exact completion on a regular category in [CV98,Hyl82] by taking only those equivalence relations obtained by comprehension from the propositional fibration. But we ended up just in a list-arithmetic pretopos, for example not necessarily closed under dependent products, since mTT is predicative and the axiom of choice is not a theorem there.

Acknowledgements: I thank Giovanni Curi, Ferruccio Guidi, Pino Rosolini, Giovanni Sambin, Thomas Streicher, Silvio Valentini for very useful discussions on the topics treated here, and Jesper Carlström about the notion of $U_{b}$.

\section{References}

[BCP03] G. Barthes, V. Capretta, and O. Pons. Setoids in type theory. J. Funct. Programming, 13(2):261-293, 2003. Special issue on "Logical frameworks and metalanguages".

[Bis67] E. Bishop. Foundations of Constructive Analysis. McGraw-Hill Book Co., 1967.

[Car95] A. Carboni. Some free constructions in realizability and proof theory. J. Pure Appl. Algebra, 103:117-148, 1995.

[Car03] J. Carlström. Subsets, quotients and partial functions in Martin-Löf's type theory. In Types for proofs and programs, volume 2646 of Lecture Notes in Comput. Sci. Springer, Berlin, 2003.

[Car04] J. Carlström. EM + Ext- + ACint is equivalent to ACext. Mathematical Logic Quarterly, 50(3):236-240, 2004.

[CC82] A. Carboni and R. Celia Magno. The free exact category on a left exact one. Journal of Australian Math. Soc., 33:295-301, 1982.

[CR00] A. Carboni and G. Rosolini. Locally cartesian closed exact completions. J. Pure Appl. Algebra, pages 103-116, 2000. Category theory and its applications (Montreal, QC, 1997).

[CV98] A. Carboni and E.M. Vitale. Regular and exact completions. Journal of Pure and Applied Algebra, 125:79-116, 1998.

[dB91] N.G. de Bruijn. Telescopic mapping in typed lambda calculus. Information and Computation, 91:189-204, 1991.

[Hof94] M. Hofmann. On the interpretation of type theory in locally cartesian closed categories. In Proceedings of CSL'94, September 1994.

[Hof97] M. Hofmann. Extensional Constructs in Intensional Type Theory. Distinguished Dissertations. Springer, 1997.

[Hyl82] J. M. E. Hyland. The effective topos. In The L.E.J. Brouwer Centenary Symposium (Noordwijkerhout, 1981), volume 110 of Stud. Logic Foundations Math., pages 165-216. North-Holland, Amsterdam-New York,, 1982.

[Jac99] B. Jacobs. Categorical Logic and Type Theory., volume 141 of Studies in Logic. Elsevier, 1999. 
[Joh02] P. T. Johnstone. Sketches of an Elephant: a Topos Theory Compendium. Vol. 2., volume 43 of Oxford Logic Guides. The Clarendon Press, Oxford University Press, New York,, 2002.

[Mai99] M. E. Maietti. About effective quotients in constructive type theory. In W. Naraschewski T. Altenkirch and B. Reus, editors, Types for proofs and programs. International workshop, TYPES '98. Kloster Irsee, Germany, March 27-31. 1999, volume 1657 of Lectures Notes in Computer Science, pages 164178. Springer Verlag, 1999.

[Mai05] M.E. Maietti. Modular correspondence between dependent type theories and categories including pretopoi and topoi. Mathematical Structures in Computer Science, 15(6):1089-1149, 2005.

[Mar84] P. Martin-Löf. Intuitionistic Type Theory, notes by G. Sambin of a series of lectures given in Padua, June 1980. Bibliopolis, Naples, 1984.

[Mar85] P. Martin Löf. On the meanings of the logical constants and the justifications of the logical laws. In Proceedings of the conference on mathematical logic, volume 2, pages 203-281. Univ. Siena, Siena, 1985. Reprinted in Nordic J. Philos. Logic, 1(1):11-60, 1996.

[ML06] P. Martin-Löf. 100 years of Zermelo's axiom of choice:what was the problem with it? The Computer Journal, 49(3):10-37, 2006.

[MS05] M.E. Maietti and G. Sambin. Toward a minimalist foundation for constructive mathematics. In L. Crosilla and P. Schuster, editor, From Sets and Types to Topology and Analysis: Practicable Foundations for Constructive Mathematics, number 48 in Oxford Logic Guides, pages 91-114. Oxford University Press, 2005.

[MV99] M.E. Maietti and S. Valentini. Can you add powersets to Martin-Löf intuitionistic type theory? Mathematical Logic Quarterly, 45:521-532, 1999.

[NPS90] B. Nordström, K. Petersson, and J. Smith. Programming in Martin Löf's Type Theory. Clarendon Press, Oxford, 1990.

[Pal05] E. Palmgren. Bishop's set theory. Slides for lecture at the TYPES summer school, 2005.

[Sam03] G. Sambin. Some points in formal topology. Theoretical Computer Science, 2003.

[See83] R. A. G. Seely. Hyperdoctrines, natural deduction and the Beck condition. Zeitschr. f. Math. Logik. und Grundlagen d. Math., 29:505-542, 1983.

[SV98] G. Sambin and S. Valentini. Building up a toolbox for Martin-Löf's type theory: subset theory. In G. Sambin and J. Smith, editors, Twenty-five years of constructive type theory, Proceedings of a Congress held in Venice, October 1995, pages 221-244. Oxford U. P., 1998.

\section{Appendix: The qmTT typed calculus}

We present here the inference rules to form sets and propositions in qmTT. Note that to write the elimination constructors of the various sets and propositions we adopt the higher order syntax as in [NPS90] ${ }^{3}$.

\footnotetext{
${ }^{3}$ For example, note that the elimination constructor of disjunction $E l_{\vee}\left(w, a_{B}, a_{C}\right)$ binds the open terms $a_{B}(x) \in A[x \in B]$ and $a_{C}(y) \in A[y \in C]$. Hence these open terms should be then encoded into the elimination constructor given that they
} 
For brevity, in presenting formal rules we omit the corresponding equality rules that are defined as in [Mar84].

The contexts are generated by the same rules as for mTT in [MS05]. Note that the piece of context common to all judgements involved in a rule is omitted and that the typed variables appearing in a context are meant to be added to the implicit context as the last one. We also recall that the contexts are made of assumptions on sets only, and that we have the rule prop-into-set and propmono mentioned in section 2 .

The rules to generate the propositions in qmTT are the following:

\section{Falsum}

F-Fs $) \perp$ prop $\quad$ E-Fs) $\frac{a \in \perp A \text { prop }}{r_{\mathrm{o}}(a) \in A}$

Propositional Equality

Eq) $\left.\left.\frac{C \text { set } c \in C \quad d \in C}{\mathrm{Eq}(C, c, d) \text { prop }} \quad \mathrm{I}-\mathrm{Eq}\right) \frac{c \in C}{\operatorname{eqc}(c) \in \mathrm{Eq}(C, c, c)} \quad \mathrm{E}-\mathrm{Eq}\right) \frac{p \in \mathrm{Eq}(C, c, d)}{c=d \in C}$

Implication

F-Im $\frac{B \text { prop } C \text { prop }}{B \rightarrow C \text { prop }} \quad \operatorname{I-Im} \frac{B \text { prop } C \text { prop } c(x) \in C[x \in B]}{\lambda_{\rightarrow} x^{B} \cdot c(x) \in B \rightarrow C}$

E-Im $\frac{b \in B \quad f \in B \rightarrow C}{\operatorname{Ap}_{\rightarrow}(f, b) \in C}$

Conjunction

F-^) $\left.\frac{B \text { prop } C \text { prop }}{B \wedge C \text { prop }} \quad \mathrm{I}-\wedge\right) \frac{B \text { prop } C \text { prop } b \in B \quad c \in C}{\langle b, \wedge c\rangle \in B \wedge C}$
$\mathrm{E}_{\mathbf{1}-\wedge} \frac{d \in B \wedge C}{\pi_{1}^{B}(d) \in B} \quad \mathrm{E}_{\mathbf{2}} \wedge \wedge \frac{d \in B \wedge C}{\pi_{2}^{C}(d) \in C}$

\section{Disjunction}

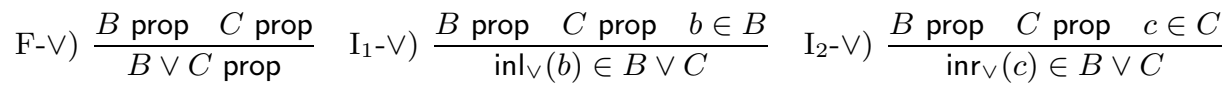

E- $) \frac{A \text { prop } \quad w \in B \vee C \quad a_{B}(x) \in A[x \in B] \quad a_{C}(y) \in A[y \in C]}{E l_{\vee}\left(w, a_{B}, a_{C}\right) \in A}$

Existential quantification

F- $\exists) \frac{C(x) \text { prop }[x \in B]}{\exists_{x \in B} C(x) \text { prop }} \quad$ I- $\left.\exists\right) \frac{C(x) \text { prop }[x \in B] \quad b \in B \quad c \in C(b)}{\langle b, \exists c\rangle \in \exists_{x \in B} C(x)}$

E- $\exists) \frac{M \text { prop } \quad d \in \exists_{x \in B} C(x) \quad m(x, y) \in M[x \in B, y \in C(x)]}{E l_{\exists}(d, m) \in M}$

are needed in the disjunction conversion rules. To simplify the notation we use the higher order syntax as in [NPS90]. Thanks to this syntax from the open term $a_{B}(x) \in$ $A[x \in B]$ we get $(x \in B) a_{B}(x)$ of higher type $(x \in B) A$. Then by $\eta$-conversion among higher types $(x \in B) a_{B}(x)$ is equal to $a_{B}$ and we can simply write the short expression $a_{B}$ to recall the open term where it comes from. 


\section{Universal quantification}

F- $\forall \frac{C(x) \text { prop }[x \in B]}{\forall_{x \in B} C(x) \text { prop }} \quad \mathrm{I}-\forall \frac{C(x) \text { prop }[x \in B] \quad c(x) \in C(x)[x \in B]}{\lambda_{\forall} x^{B} \cdot c(x) \in \forall_{x \in B} C(x)}$
$\mathrm{E}-\forall \frac{b \in B \quad f \in \forall_{x \in B} C(x)}{\operatorname{Ap}_{\forall}(f, b) \in C(b)}$

The rules to generate qmTT sets are the following:

Empty set

F-Em) $N_{0}$ set $\quad$ E-Em) $\frac{a \in N_{0} \quad A(x) \text { set }\left[x \in N_{0}\right]}{\operatorname{empo}_{\circ}(a) \in A(a)}$

\section{Singleton set}
S) $N_{1}$ set
$\mathrm{I}-\mathrm{S}) \star \in \mathrm{N}_{1}$
C-S) $\frac{t \in \mathrm{N}_{1} \quad M(z)\left[z \in \mathrm{N}_{1}\right] \quad c \in M(\star)}{E l_{\mathrm{N}_{1}}(t, c) \in M(t)}$

Indexed Sum set
$\mathrm{F}-\Sigma) \frac{C(x) \text { set }[x \in B]}{\Sigma_{x \in B} C(x) \text { set }}$
$\mathrm{I}-\Sigma) \frac{b \in B \quad c \in C(b)}{\langle b, c\rangle \in \Sigma_{x \in B} C(x)}$ $M(z)\left[z \in \Sigma_{x \in B} C(x)\right]$
$\mathrm{E}-\Sigma) \frac{d \in \Sigma_{x \in B} C(x) \quad m(x, y) \in M(\langle x, y\rangle)[x \in B, y \in C(x)]}{E l_{\Sigma}(d, m) \in M(d)}$ $M(z)\left[z \in \Sigma_{x \in B} C(x)\right]$
$\mathrm{C}-\Sigma) \frac{b \in B \quad c \in C(b) \quad m(x, y) \in M(\langle x, y\rangle)[x \in B, y \in C(x)]}{E l_{\Sigma}(\langle b, c\rangle, m)=m(b, c) \in M(\langle b, c\rangle)}$

Disjoint Sum set

$$
\begin{aligned}
& \text { +) } \left.\frac{C \text { set } B \text { set }}{C+B \text { set }} \quad \mathrm{I}_{1-+}+\frac{c \in C}{\operatorname{inl}(c) \in C+B} \quad \mathrm{I}_{2-}+\right) \frac{b \in B}{\operatorname{inr}(b) \in C+B} \\
& A(z)[z \in C+B] \\
& \mathrm{E}-+) \frac{w \in C+B \quad a_{C}(x) \in A(\operatorname{inl}(x))[x \in C] \quad a_{B}(y) \in A(\operatorname{inr}(y))[y \in B]}{E l_{+}\left(w, a_{C}, a_{B}\right) \in A(w)} \\
& A(z)[z \in C+B] \\
& \left.\mathrm{C}_{1-+}\right) \frac{c \in C \quad a_{C}(x) \in A(\operatorname{inl}(x))[x \in C] \quad a_{B}(y) \in A(\operatorname{inr}(y))[y \in B]}{E l_{+}\left(\operatorname{inl}(c), a_{C}, a_{B}\right)=a_{C}(c) \in A(\operatorname{inl}(c))} \\
& A(z)[z \in C+B] \\
& \left.\mathrm{C}_{2^{-}}+\right) \frac{b \in B \quad a_{C}(x) \in A(\operatorname{inl}(x))[x \in C] \quad a_{B}(y) \in A(\operatorname{inr}(y))[y \in B]}{E l_{+}\left(\operatorname{inr}(b), a_{C}, a_{B}\right)=a_{B}(b) \in A(\operatorname{inr}(b))}
\end{aligned}
$$

\section{Dependent Product set}

$$
\begin{array}{ll}
\text { F- } \Pi \frac{C(x) \text { set }[x \in B]}{\Pi_{x \in B} C(x) \text { set }} & \text { I- } \Pi \frac{c \in C(x)[x \in B]}{\lambda x^{B} \cdot c \in \Pi_{x \in B} C(x)} \\
\text { E- } \Pi \frac{b \in B \quad f \in \Pi_{x \in B} C(x)}{\operatorname{Ap}(f, b) \in C(b)} & \beta \mathrm{C}-\Pi \frac{b \in B \quad c(x) \in C(x)[x \in B]}{\operatorname{Ap}\left(\lambda x^{B} \cdot c(x), b\right)=c(b) \in C(b)} \\
\beta \mathrm{C}-\Pi \frac{c \in \Pi_{x \in B} C(x)}{\lambda x^{B} \cdot \operatorname{Ap}(c, x)=c \in \Pi_{x \in B} C(x)} &
\end{array}
$$




\section{Boolean universe}

F-bU $U_{b}$ set $\quad \mathrm{I}_{1}$-bU $\widetilde{N_{0}} \in U_{b} \quad \mathrm{I}_{2}$-bU $\widetilde{N_{1}} \in U_{b}$

E-bU $\frac{d \in U_{b}}{T(d) \text { set }} \quad \beta \mathrm{C}_{1}$-bU $T\left(\widetilde{N_{0}}\right)=N_{0} \quad \beta \mathrm{C}_{2}$-bU $\quad T\left(\widetilde{N_{1}}\right)=N_{1}$

\section{Quotient set}

$$
\begin{aligned}
& \operatorname{refl}(x) \in R(x, x)[x \in A] \\
& R(x, y) \text { prop }[x \in A, y \in A] \quad \operatorname{sym}(x, y, z) \in R(y, x)[x \in A, y \in A, z \in R(x, y)] \\
& \operatorname{trans}(x, y, z, u, v) \in R(x, z)[x \in A, y \in A, z \in A \\
& \text { Q) } \\
& A / R \text { set } \\
& \text { I-Q) } \left.\frac{a \in A A / R \text { set }}{[a] \in A / R} \quad \text { eq- } Q\right) \frac{a \in A \quad b \in A \quad d \in R(a, b) A / R \text { set }}{[a]=[b] \in A / R} \\
& L(z)[z \in A / R] \\
& \text { E-Q) } \frac{p \in A / R \quad l(x) \in L([x])[x \in A] \quad l(x)=l(y) \in L([x])[x \in A, y \in A, d \in R(x, y)]}{E l_{Q}(l, p) \in L(p)} \\
& L(z)[z \in A / R] \\
& \mathrm{C}-\mathrm{Q}) \frac{a \in A \quad l(x) \in L([x])[x \in A] \quad l(x)=l(y) \in L([x])[x \in A, y \in A, d \in R(x, y)]}{E l_{Q}(l,[a])=l(a) \in L([a])}
\end{aligned}
$$

\section{Effectiveness}

$$
\frac{a \in A \quad b \in A \quad[a]=[b] \in A / R}{\operatorname{eff}(a, b) \in R(a, b)}
$$

\section{List set}

$$
\begin{aligned}
& \text { list) } \left.\frac{C \text { set }}{\operatorname{List}(C) \text { set }} \quad \mathrm{I}_{1} \text {-list }\right) \epsilon \in \operatorname{List}(C) \quad \mathrm{I}_{2} \text {-list) } \frac{s \in \operatorname{List}(C) \quad c \in C}{\operatorname{cons}(s, c) \in \operatorname{List}(C)} \\
& L(z)[z \in \operatorname{List}(C)] \\
& \text { E-list) } \frac{s \in \operatorname{List}(C) \quad a \in L(\epsilon) \quad l(x, y, z) \in L(\operatorname{cons}(x, y))[x \in \operatorname{List}(C), y \in C, z \in L(x)]}{E l_{\text {List }}(a, l, s) \in L(s)} \\
& L(z)[z \in \operatorname{List}(C)] \\
& \left.\mathrm{C}_{1} \text {-list }\right) \frac{a \in L(\epsilon) \quad l(x, y, z) \in L(\operatorname{cons}(x, y))[x \in L i s t(C), y \in C, z \in L(x)]}{E l_{\text {List }}(a, l, \epsilon)=a \in L(\epsilon)} \\
& L(z)[z \in \operatorname{List}(C)] \\
& s \in \operatorname{List}(C) \quad c \in C \quad a \in L(\epsilon) \\
& \left.\mathrm{C}_{2} \text {-list }\right) \frac{l(x, y, z) \in L(\operatorname{cons}(x, y))[x \in \operatorname{List}(C), y \in C, z \in L(x)]}{E l_{\text {List }}(a, l, \operatorname{cons}(s, c))=l\left(s, c, E l_{\text {List }}(a, l, s)\right) \in L(\operatorname{cons}(s, c))}
\end{aligned}
$$

Note that $\operatorname{List}\left(N_{1}\right)$ corresponds to the set of natural numbers represented as lists on a singleton, with $0 \equiv \epsilon$ and $s(n) \equiv \operatorname{cons}(n, *)$ for $n \in \operatorname{List}\left(N_{1}\right)$. 